\title{
Calcificación de la arteria facial como hallazgo radiográfico: Reporte de 6 casos y revisión de la literatura
}

Calcification of the facial artery as a radiographic finding: Report of 6 cases and review of the literature

\author{
Fernando Russbelts Sthorayca Retamozo ${ }^{1, a, c}$, Vilma Elizabeth Ruiz García de Chacón ${ }^{1, a, b, d}$
}

\section{RESUMEN}

La calcificación de la arteria facial o arteriosclerosis de Monckeberg se caracteriza por la calcificación distrófica de la túnica media de la arteria sin afectar a otras túnicas ni obstruir la luz del vaso, por lo tanto, no produce sintomatología. Su etiología aún es desconocida; sin embargo, la evidencia científica reporta que se presenta con mayor frecuencia en pacientes de edad avanzada con diabetes mellitus o enfermedad renal crónica. Generalmente se identifica como hallazgo radiográfico sutil en imágenes maxilofaciales (radiografías panorámicas o periapicales posteriores). El objetivo de este artículo es reportar 6 casos de hallazgo radiográfico de calcificación de la arteria facial, con el fin que el odontólogo reconozca esta entidad y su fuerte asociación con enfermedades sistémicas de tal manera que el paciente pueda ser derivado de forma oportuna a un médico especialista y ser tratado adecuadamente.

PALABRAS CLAVE: Calcificación vascular, arteriosclerosis, radiología.

\section{SUMMARY}

Calcification of the facial artery or Monckeberg's arteriosclerosis is characterized by the dystrophic calcification of the middle tunic of the artery without affecting other tunics or obstructing the lumen of the vessel, therefore, it does not produce symptoms. Its etiology is still unknown; however, the scientific evidence reports that occur more frequently in elderly patients with diabetes mellitus or chronic kidney disease. It is usually identified as a radiographic finding that appears subtly on maxillofacial images (panoramic or periapical posterior radiographs). The objective of this article is report 6 cases of radiographic finding of calcification of the facial artery, so that the dentist can recognizes this entity and its strong association with systemic diseases so that the patient can be referred opportunely to a specialist and be treated adequately.

KEY WORD: Vascular calcification, arteriosclerosis, radiology.

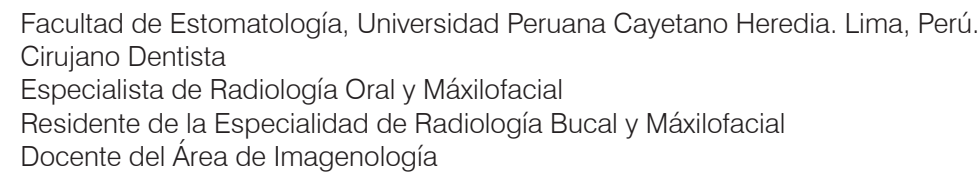




\section{INTRODUCCIÓN}

La calcificación es un proceso bioquímico caracterizado por el depósito de sales de calcio que ocurre de manera natural en tejidos óseos y dentarios. Cuando este proceso se altera y se manifiesta en tejidos blandos se denomina calcificaciones patológicas o heterotópicas; según la literatura existen tres tipos de calcificaciones patológicas: distróficas, idiopáticas y metastásicas, estando la calcificación vascular dentro del tipo de calcificaciones distróficas $(1,2)$.

La calcificación vascular es la deposición de fosfato de calcio que puede manifestarse en vasos sanguíneos y en las válvulas cardiacas como consecuencia del envejecimiento, siendo más frecuente en pacientes con diabetes, dislipemia, enfermedades genéticas y enfermedades que involucran alteraciones del metabolismo de calcio $(3,4)$. Actualmente existen dos tipos de calcificación arterial: la aterosclerosis y la arteriosclerosis de Monckeberg $(5,6)$.

La aterosclerosis está asociada a la calcificación de la túnica intima de la arteria y se caracteriza por la acumulación de lípidos, tejidos fibrosos y depósitos de calcio en las paredes de las arterias $(3,7,8)$; mientras que la arteriosclerosis de Monckeberg está asociada a la calcificación de la túnica media y se caracteriza por la degeneración y perdida de elasticidad de las fibras arteriales; así mismo, estos depósitos de calcio no están relacionados con el estrechamiento de la luz del vaso o interrupción de la túnica intima $(3,5,9)$. La etiología de la calcificación de Monckeberg sigue sin estar clara; sin embargo, se relaciona a pacientes con diabetes mellitus o enfermedad renal crónica $(5,10,11)$.

La mayoría de los pacientes que presentan este tipo de calcificación son asintomáticos, y generalmente se identifican por hallazgo radiográfico (radiografías panorámicas o periapicales posteriores), presentándose como un par de líneas radiopacas paralelas, que pueden tener un trayecto lineal o un camino tortuoso y se denomina como "tubería", "vía de tranvía" o "seguimiento de riel" $(2,3)$.

El objetivo de este artículo es reportar 6 casos de hallazgo radiográfico de calcificación de la arteria facial ya que es fundamental que el odontólogo identifique esta rara condición en los exámenes radiográficos y así el paciente pueda ser derivado a un médico especialista con el fin de minimizar el riesgo sistémico.

\section{Reporte de casos}

Todos los casos reportados corresponden a pacientes que acudieron al Servicio de Radiología

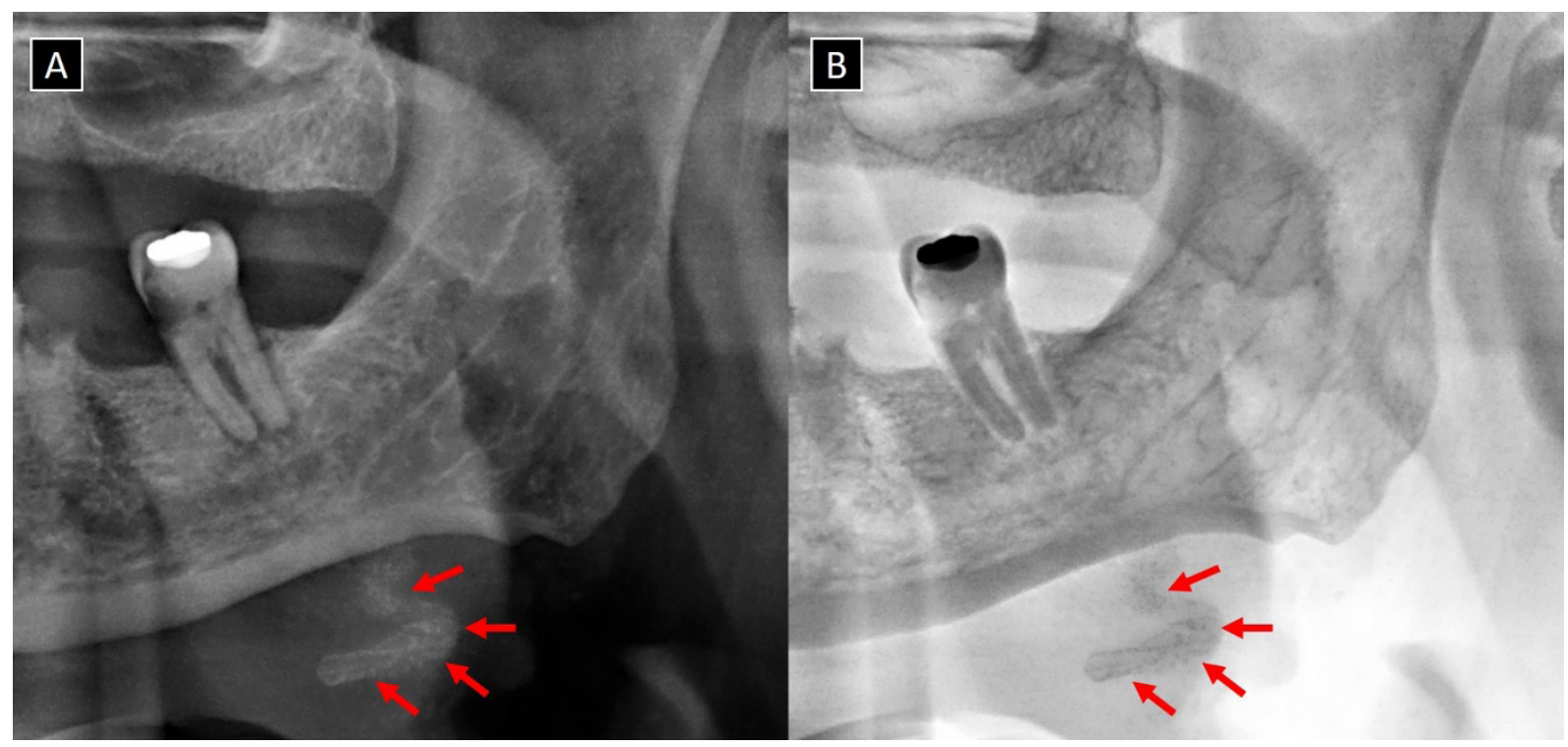

Figura 1. (A y B). Radiografía panorámica recortada donde se observa una imagen radiopaca de camino tortuoso proyectada en tejidos blandos a nivel caudal de la región antegonial de lado izquierdo, signos radiográficos compatible con calcificación de la arteria facial. 
Bucal y Maxilofacial del Centro Dental Docente, Facultad de Estomatología de la Universidad Peruana Cayetano Heredia como parte de su tratamiento integral.

Caso 1: Paciente de sexo masculino de 38 años de edad (Figura 1).

Caso 2: Paciente de sexo masculino de 64 años de edad (Figura 2).
Caso 3: Paciente de sexo masculino de 81 años de edad (Figura 3).

Caso 4: Paciente de sexo masculino de 75 años de edad (Figura 4).

Caso 5: Paciente de sexo femenino de 83 años de edad (Figura 5).

Caso 6: Paciente de sexo masculino de 79 años de edad (Figura 6).

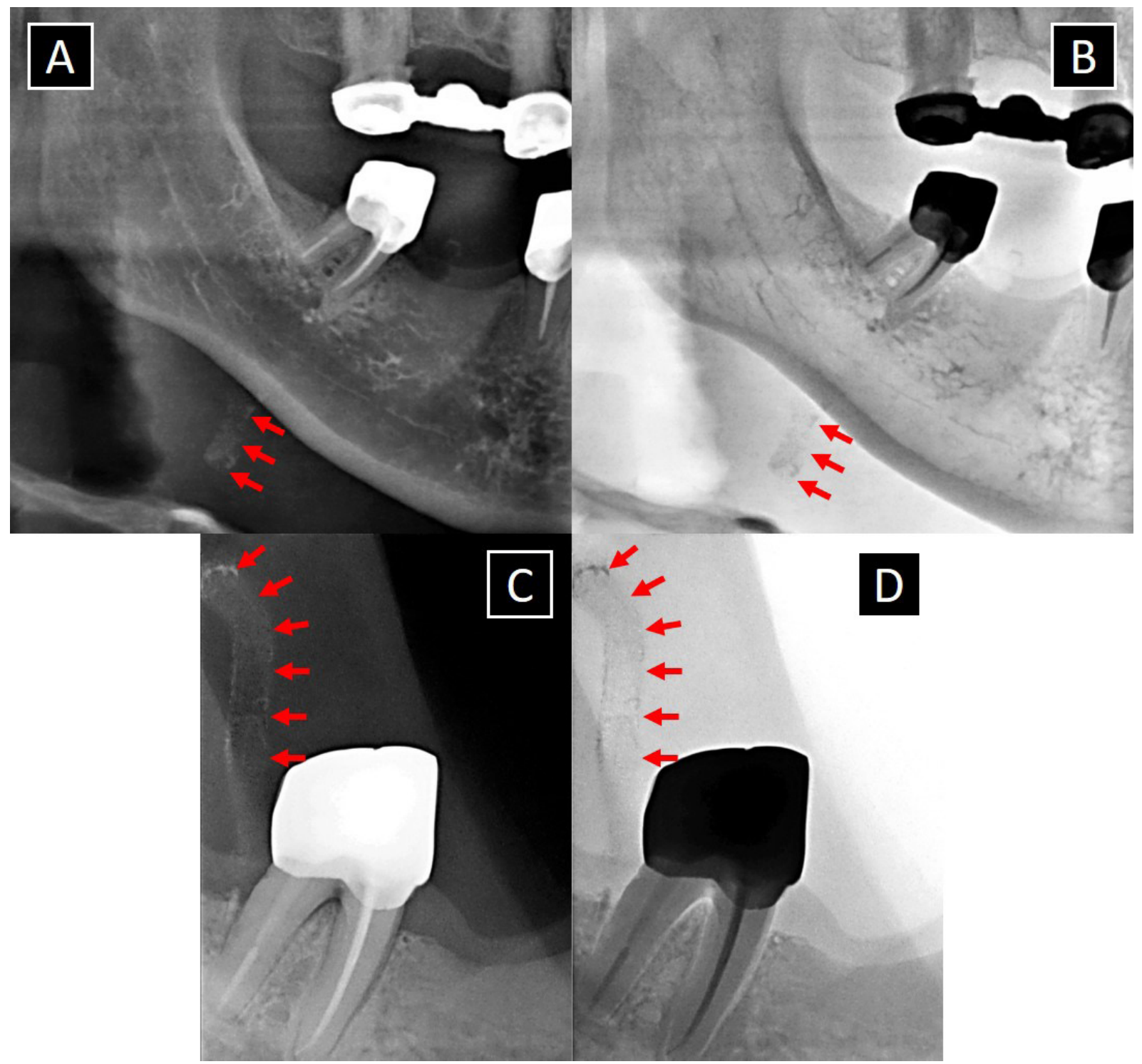

Figura 2. (A y B). Radiografía panorámica recortada donde se observa un par de líneas radiopacas paralelas de trayecto recto proyectado en tejidos blandos de a nivel caudal del cuerpo mandibular derecho, signos radiográficos compatible con calcificación de la arteria facial. (C y D) Radiografía periapical del mismo paciente donde muestra la arteriosclerosis con un patrón de vías de ferrocarril proyectado a nivel cefálico-distal de la pieza 46. 


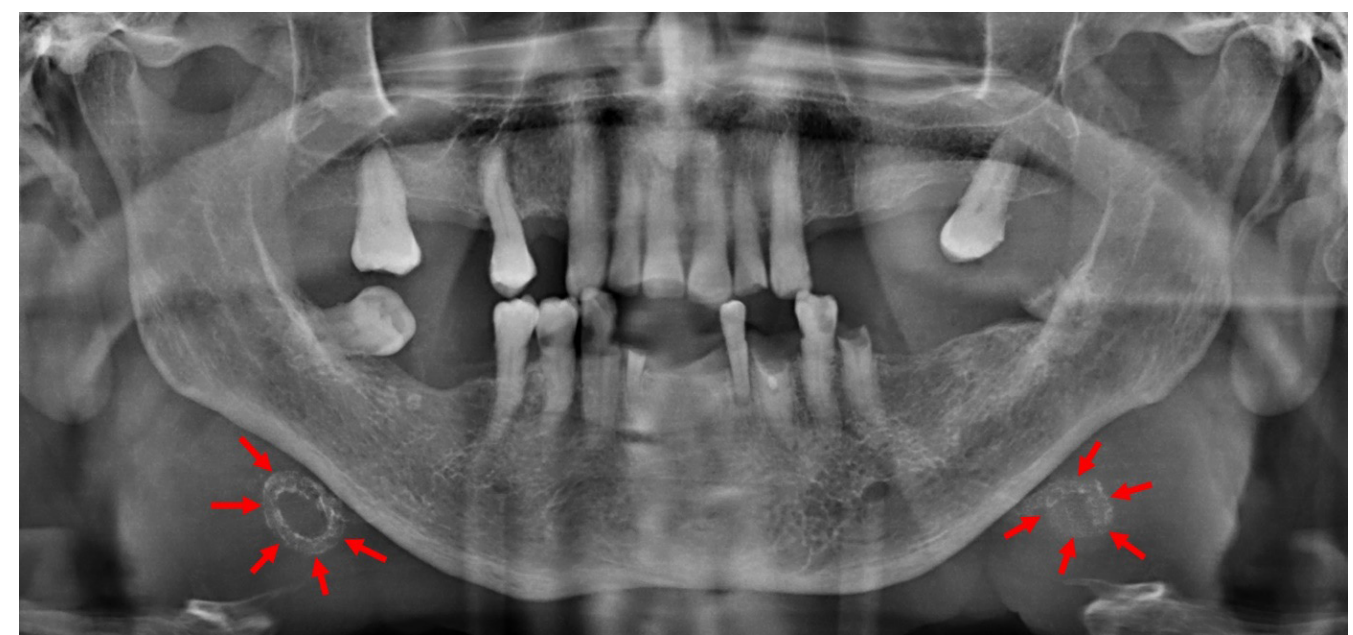

Figura 3. Radiografía panorámica donde muestra la presencia de dos imágenes radiopacas de camino tortuoso proyectados en tejidos blandos a nivel caudal del cuerpo mandibular derecho e izquierdo, signos radiográficos compatible con calcificación de la arteria facial.

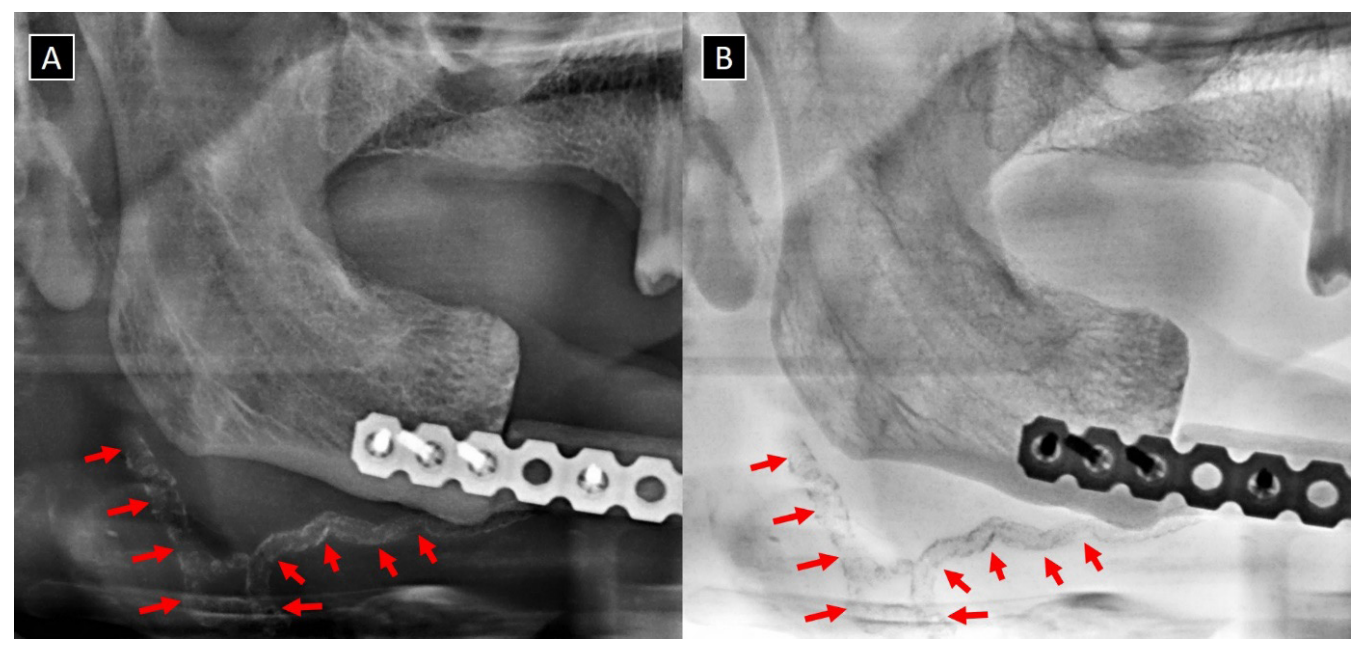

Figura 4. (A y B). Radiografía panorámica recortada donde muestra una imagen radiopaca de camino tortuoso proyectado en tejidos blandos que se extiende en dirección horizontal desde el ángulo mandibular derecho, bifurcándose a nivel antegonial hasta el cuerpo mandibular derecho, signos radiográficos de arteriosclerosis.

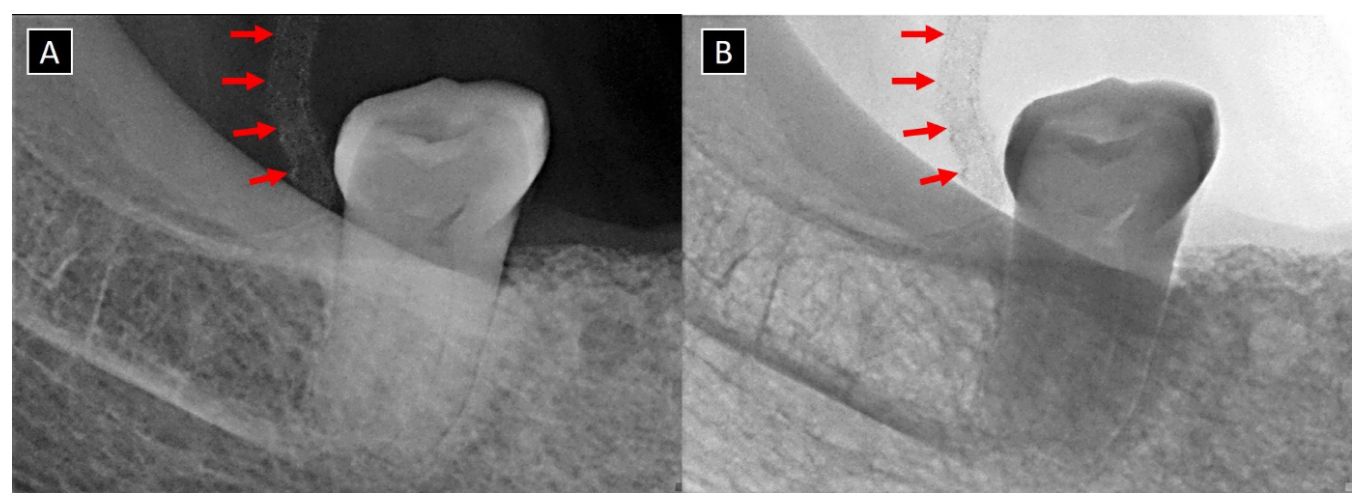

Figura 5. (Ay B). Radiografía periapical donde de observa un par de líneas radiopacas paralelas de trayecto recto proyectado en tejidos blandos a nivel cefálico-distal de la pieza 47 que se asemeja a un patrón de vías de ferrocarril, signos radiográficos de arteriosclerosis. 


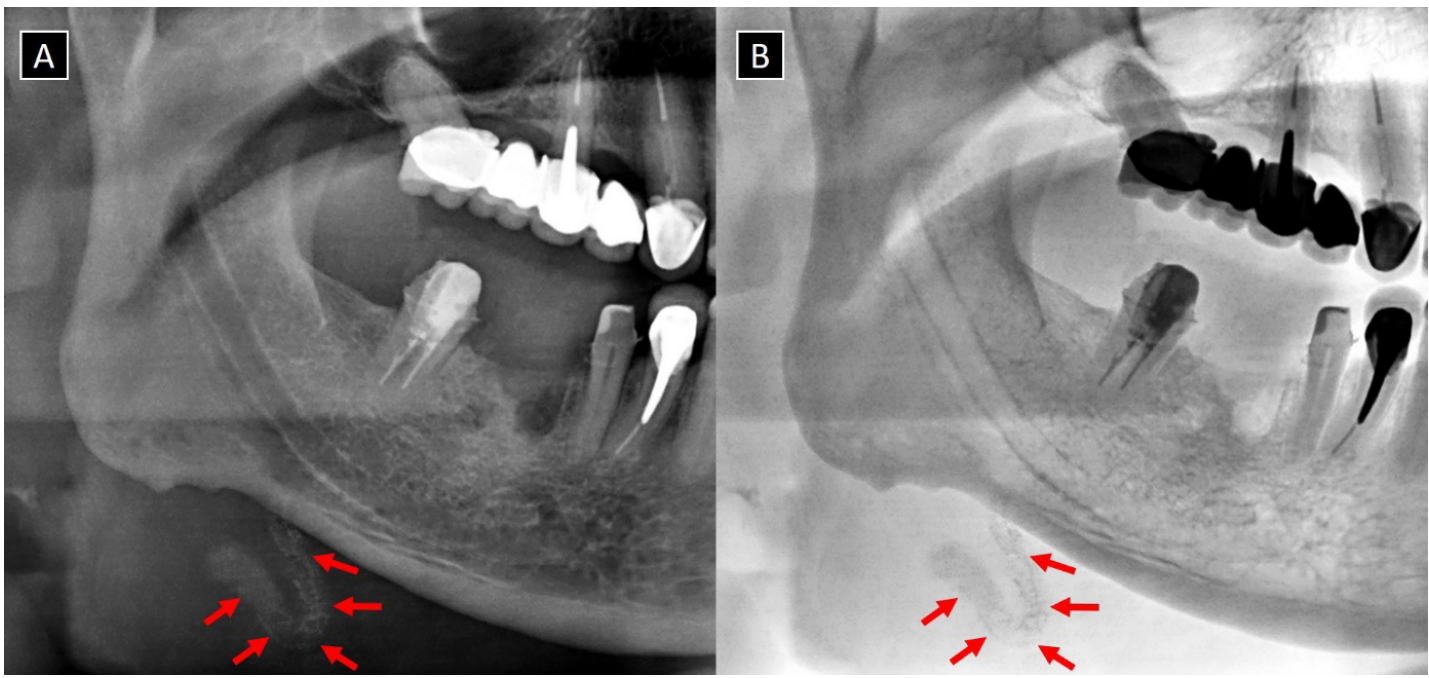

Figura 6. (A y B). Radiografía panorámica recortada donde muestra la presencia de una imagen radiopaca de camino tortuoso proyectado en tejidos blandos a nivel caudal de la región antegonial del lado derecho, signos radiográficos compatible con calcificación de la arterial facial.

\section{DISCUSIÓN}

La calcificación vascular se puede presentar en cualquier región del sistema cardiovascular, y se manifiesta tanto en la túnica íntima como en la túnica media de los vasos sanguíneos (3). La calcificación arterial de la túnica intima es parte del proceso aterosclerótico caracterizándose por la formación de placas calcificadas dentro de la misma; sin embargo, la túnica media está asociada a la arteriosclerosis de Monckeberg y se caracteriza por la deposición de calcio dentro de ella disminuyendo su elasticidad de las arterias, sin afectar a otras túnicas vasculares ni estrechar la luz del vaso $(5,11,12)$.

La arteriosclerosis de Monckeberg fue descrita en 1903 por el patólogo alemán Johann Monckeberg; otros nombres encontrados en la literatura son esclerosis de Monckeberg y calcinosis de Monckeberg. Esta entidad está fuertemente asociada a la enfermedad renal crónica y a la diabetes mellitus $(4,6,13)$. La ecografía es un método de imagen no invasivo e indoloro que permite determinar el diagnóstico definitivo y la localización exacta de este tipo de calcificación $(4,9)$. En cuanto al diagnóstico diferencial encontramos la calcificación de la arteria carótida que se presenta como imágenes radiopacas paralelas y curvilíneas proyectadas en tejidos blandos a nivel de la tercera y cuarta vértebra cervical, por debajo o lateral del hueso hioides (3). Generalmente la arteriosclerosis es asintomática, pero en etapa avanzada se puede manifestar signos patológicos tales como la enfermedad vascular periférica, gangrena cutánea y miositis como resultado de la insuficiencia vascular (2). Se reporta que el $94 \%$ de pacientes con diabetes mellitus después de los 35 años de enfermedad presentan calcificación arterial; siendo más frecuente en pacientes ancianos (14). Este hecho coincide con los casos presentados ya que de los 6 casos reportados 4 pacientes son mayores de 64 años.

Otros estudios como el de Kroger et al. analizaron 4814 pacientes entre 45 a 75 años de edad, desde el año 2000 al 2003 obteniendo como resultado que este tipo de calcificación es más frecuente en hombres $(13.3 \%)$ que en mujeres (6.9\%) (15). Estos resultados coinciden con los casos reportados ya que de los 6 pacientes; 5 son del sexo masculino y solo 1 es del sexo femenino. Tahmasbi et al. reportaron un caso de un hombre de 65 años que tenía cáncer nasofaríngeo, enfermedad renal en etapa terminal, diabetes mellitus e hiperparatiroidismo; al examen imagenológico presentaba calcificación bilateral de la arteria facial (arterioesclerosis) y a su vez también presentaba calcificación de la arteria carótida (aterosclerosis) (3). Este artículo coincide con nuestro reporte de caso número 3 , reportando un paciente de sexo masculino de 81 años de edad que al examen imagenológico presenta calcificación bilateral de la 
arteria facial (Figura 3). Omami reportó otro caso de un hombre de 59 años con un historial médico de diabetes mellitus, hipertensión y trasplante de riñón que al examen imagenológico presentaba signos radiográficos característicos de arteriosclerosis con una configuración de vía de tranvía (5). Este reporte de caso coincide con el caso número 4 , reportando un paciente masculino de 75 años de edad que al examen radiográfico muestra la calcificación con el mismo patrón imagenológico (arteriosclerosis) (Figura 4).

La etiología de esta enfermedad aún se desconoce, aunque Monckeberg inicialmente describe que la calcificación de la túnica media afecta principalmente a las arterias de las extremidades inferiores y que en ocasiones afecta a las arterias de las extremidades superiores $(3,5,11)$.

Esta enfermedad generalmente es un hallazgo incidental y puede ser identificado clínicamente o radiográficamente. En cuanto al examen clínico (a la palpación de la zona afectada) puede ser descrito como "vástago de tubo" y se presenta de manera asintomática, también se puede manifestar de forma dura y sin pulso, aunque hay situaciones en la que se puede percibir un pulso delimitado al final de la zona calcificada (16). En cuanto a lo radiográfico se descubre mediante imágenes rutinarias (11), presentándose como un par de líneas radiopacas paralelas entre sí, que pueden tener un curso recto o un camino tortuoso mostrando un patrón que asemeja a las vías del ferrocarril, proyectándose a nivel de tejidos blandos del cuello y que pueden ser descritas como "vástago de tubería", "vía de tranvía" o "vía de ferrocarril" $(2,13)$.

En el área de radiología bucal y maxilofacial es común encontrar calcificaciones de tejidos blandos que pueden ser de origen fisiológico o patológico, es por ello que el radiólogo maxilofacial debe hacer una revisión e interpretación exhaustiva de los exámenes radiográficos rutinarios (5). Los profesionales de salud oral deben de estar al tanto de este tipo de calcificación arterial y su hallazgo incidental en radiografías panorámicas y periapicales, ya que por su fuerte asociación con enfermedades sistémicas es un signo de alarma; de tal forma que los pacientes puedan ser derivados de manera oportuna al médico especialista y ser tratados adecuadamente.

\section{Correspondencia:}

Fernando Russbelts Sthorayca Retamozo

Calle Bolívar 434. Tacna-Perú.

Correo electrónico: fernando.sthorayca@upch.pe

\section{REFERENCIAS BIBLIOGRÁFICAS}

1. Herrera R, Agurto A, Diaz L, Gonzales H. Radiología digital en la evaluación de calcificaciones en tejidos blandos. Kiru. 2012;9(2):161-6.

2. Cueva Y. frecuencia de ateromas calcificados de arteria carótida en radiografías panorámicas digitales de la Universidad Peruana Cayetano Heredia. Lima, 2011-2015. Tesis para obtener el Título de Especialista en Radiología Bucal y Maxilo Facial. Lima, Perú: Universidad Peruana Cayetano Heredia; 2015.

3. Tahmasbi-Arashlow M, Barghan S, Kashtwari D, Nair MK. Radiographic manifestations of Mönckeberg arteriosclerosis in the head and neck region. Imaging Sci Dent. 2016;46(1):53-6.

4. Valdivieso JM. Vascular calcification: types and mechanisms. Nefrologia. 2011;31(2):142-7.

5. Omami G. Monckeberg Arteriosclerosis: A Telltale Sign. J Oral Maxillofac Surg. 2017;75(11):2383-4.

6. Adhami Firas, Ahmed A, Omami G, Mathew R. Softtissue calcification on a panoramic radiograph. J Am Dent Assoc. 2016; 147(5):362-5.

7. Borba DL, Hipólito UV, Pereira YCL. Early diagnosis of atherosclerosis with panoramic radiographs: a review. J Vasc Bras. 2016;15(4):302-7.

8. Nasseh I, Aoun G. Carotid Artery Calcification: A Digital Panoramic-Based Study. Diseases. 2018;6(1): E15.

9. Lanzer P, Boehm M, Sorribas V, Thiriet M, Janzen J, Zeller T, St Hilaire C, Shanahan C. Medial vascular calcification revisited: review and perspectives. Eur Heart J. 2014; 35(23):1515-25.

10. Amann K. Media calcification and intima calcification are distinct entities in chronic kidney disease. Clin J Am Soc Nephrol. 2008;3(6):1599-605.

11. Frazier JJ, Casian R, Benson BW. Mönckeberg medial calcinosis of the infraorbital arteries: a first case report. Oral Surg Oral Med Oral Pathol Oral Radiol. 2018; 125(2):e31-e35.

12.O'Neill WC, Lomashvili KA. Recent progress in the treatment of vascular calcification. Kidney Int. 2010;78 (12):1232-9.

13. Frazier JJ, Liang H, Fliny DJ, Benson BW. Mönckeberg medial calcinosis of the infraortital arteries. A first case report. Oral Surg Oral Med Oral Pathol Oral Radiol. 2019;127(1):35.

14. Couri CE, da-Silva GA, Martinez JA, Pereira F-de-A, de-Paula FJ. Mönckeberg's sclerosis - is the artery the only target of calcification? BMC Cardiovasc 
Disord. 2005; 12 (5): 34.

15. Kröger K, Stang A, Kondratieva J, Moebus S, Beck E, et al. Prevalence of peripheral arterial disease results of the Heinz Nixdorf recall study. Eur J Epidemiol. 2006; 21(4):279-85.
16. Castling B, Bhatia S, Ahsan F. Mönckeberg's arteriosclerosis: vascular calcification complicating microvascular surgery. Int J Oral Maxillofac Surg. 2015; 44(1):34-6.

Recibido : 13-05-2020

Aceptado : 14-11-2020 